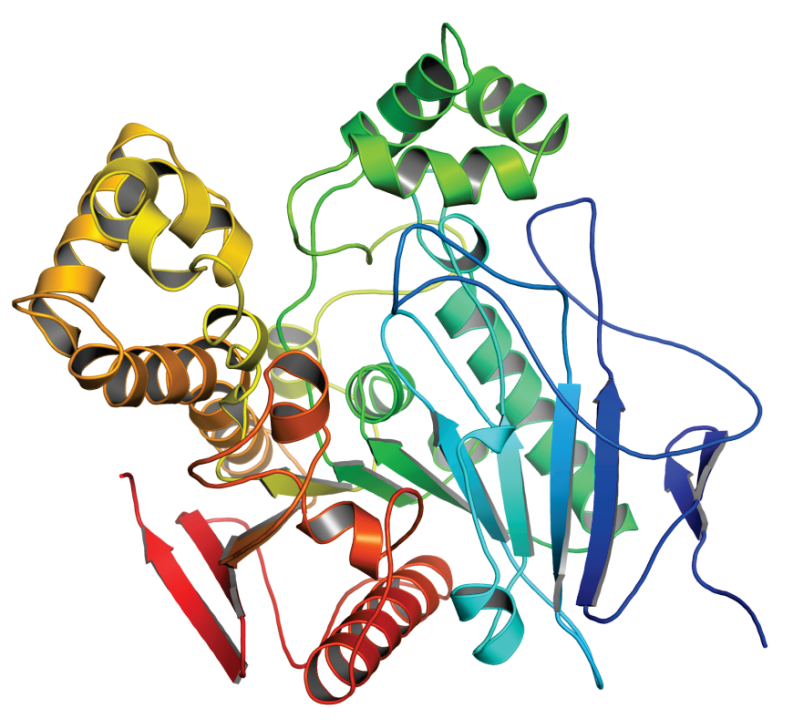

Figure 1. Structure of the first insect $\beta$-esterase solved, esterase-6

Keywords: Carboxylesterase, Protein Structure, Docking, Molecular Dynamics,

\section{MS5-P53 Structural investigation of streptococcal collagen-like protein Scl2 from invasive M3-type group A Streptococcus pyogenes}

Flavia Squeglia ${ }^{1}$, Maria Romano ${ }^{1}$, Beth Bachert ${ }^{2}$, Alfonso De Simone $^{3}$, Slawomir Lukomski ${ }^{2}$, Rita Barisio ${ }^{1}$

1. Institute of Biostructure and Bioimaging (IBB) of the Italian National Research Council (CNR)

2. Department of Microbiology, Immunology, and Cell Biology, West Virginia University School of Medicine 2095 Health Sciences North, WV 26506, Morgantown, USA

3. Imperial College London, South Kensington, London SW7 2AZ, UK

email: squegliaflavia@gmail.com

The arsenal of virulence factors deployed by streptococci includes streptococcal collagen-like (Scl) proteins. These proteins, which are characterized by a globular domain and a collagen-like domain, play key roles in streptococcal pathogenesis, like establishing host-adhesion, evade the host immune defenses, or mediate biofilm formation [1,2]. However, no three-dimensional structural information is available so far for any of the Scl proteins. In this work, we proved that the Scl2 protein is expressed by invasive M3-type strain MGAS315 of Streptococcus pyogenes and is found on the bacterial cell surface. We solved the $\mathrm{x}$-ray crystallographic structure of the globular domain of Scl2.3, the first of any Scl, and used modeling and molecular dynamics techniques to gather structural information on the entire Scl2.3. This structure shows a novel fold among collagen trimerization domains of either bacterial or human origin. Despite there being low sequence identity, we observed that Scl2.3 globular domain structurally resembles the gp41 subunit of the envelope glycoprotein from human immunodeficiency virus type 1, an essential subunit for viral fusion to human T cells. Molecular dynamics data evidence a high flexibility of Scl2.3 with remarkable inter-domain motions that are likely instrumental to its biological function $[3,4]$. Our results provide molecular tools for the understanding of Scl-mediated streptococcal pathogenesis and important structural insights for the future design of small molecular inhibitors of streptococcal invasion.

References:

1. Humtsoe J.O. et al., (2005), J Biol Chem, 280(14):13848-57;

2. Rasmussen M. \& Bjorck L., (2001), Mol Microbiol., 40(6):1427-38;

3. Squeglia et al., (2013), Acta Crystallogr Sect F Biol Cryst Commun., 69(Pt 9):1023-5;

4. Squeglia et al., (2014), J Biol Chem., 289(8):5122-33;

Keywords: Collagen-like protein, Crystal Structure, Molecular Dynamics 